EPJ Web of Conferences 41, 05039 (2013)

DOI: $10.1051 /$ epjconf/20134105039

(C) Owned by the authors, published by EDP Sciences, 2013

\title{
Ballistic energy transport in PEG oligomers
}

\author{
Zhiwei Lin, Natalia I. Rubtsova, Victor V. Kireev, Igor V. Rubtsov \\ Department of Chemistry, Tulane University, New Orleans, LA 70118, U.S.A.
}

\begin{abstract}
Energy transport between the terminal groups of the azido-PEG-succinimide ester compounds with a number of repeating PEG units of $0,4,8$, and 12 was studied using relaxation-assisted two-dimensional infrared spectroscopy. The through-bond energy transport time, evaluated as the waiting time at which the cross peak maximum is reached, $T_{\max }$, was found to be linearly dependent on the chain length for chain lengths up to $60 \AA$ suggesting a ballistic energy transport regime. The through-bond energy transport speed is found to be ca. $500 \mathrm{~m} / \mathrm{s}$. The cross-peak amplitude at the maximum decays exponentially with the chain length with a characteristic decay distance of $15.7 \pm$ $1 \AA$ A. Substantial mode delocalization across the PEG bridge is found, which can support the energy propagation as a wavepacket.
\end{abstract}

\section{Introduction}

Understanding the energy transport dynamics on a molecular scale is vital for a variety of fields including molecular electronics, nanoscience, and biochemistry. The ballistic transport regime has been previously observed experimentally in macroscopic systems at low temperatures (crystals)[1] and in mesoscopic samples, such as carbon nanotubes [2]. Both acoustic and optical phonons can transfer energy ballistically. Ballistic energy transport in molecules following electronic excitation $\left(\sim 17,000 \mathrm{~cm}^{-1}\right)$ was reported by Troe and coworkers; the transport with nearly constant velocity was observed through alkane chains of various length containing up to 6 carbon atoms [3]. A dynamic transition in a peptide helix in response to a temperature change was attributed to a switch between diffusive and ballistic energy transfer mechanisms [4]. Dlott and coworkers have found the energy transport in long-chain stretched hydrocarbons exposed to ca. $800 \mathrm{~K}$ transient temperature gradient to be ballistic in monolayers of alkanes self-assembled at a gold surface [5].
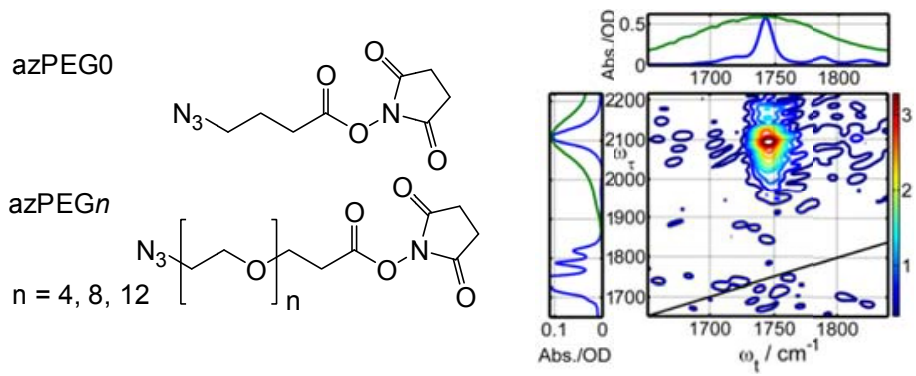

Fig. 1. Structures of the azPEG $n$ compounds and 2DIR spectrum of azPEG4 measured at $T=60 \mathrm{ps}$.

This is an Open Access article distributed under the terms of the Creative Commons Attribution License 2.0, which permits unrestricted use, distribution, and reproduction in any medium, provided the original work is properly cited. 


\section{Results}

A series of azido-PEG-succinimide ester oligomers with a number of repeating PEG units of $0,4,8$, and 12 (azPEGn, Fig. 1) was investigated using relaxation-assisted two-dimensional infrared (RA 2DIR) spectroscopy [6], which allows following the energy transport in molecules. Excitation of the azido group in the compounds at ca. $2100 \mathrm{~cm}^{-1}$ generates an excess energy which propagates in the molecule as well as dissipates into the solvent. The waiting-time, $T$, dependencies of three cross peaks, $\mathrm{N} \equiv \mathrm{N} / 1742, \mathrm{~N} \equiv \mathrm{N} / 1778$, and $\mathrm{N} \equiv \mathrm{N} / 1819$, are shown in Fig. 2 . The $\mathrm{N} \equiv \mathrm{N} / 1742$ and $\mathrm{N} \equiv \mathrm{N} / 1778$ cross-peaks are sensitive to the temperature increase in the sample induced by the $\mathrm{NN}$ mode excitation, which is apparent from the presence of plateaus at $T>50 \mathrm{ps}$ (Fig. 2, left). The mode at $1819 \mathrm{~cm}^{-1}$, which involves an almost equal mixture of the symmetric $\mathrm{C}=\mathrm{O}$ stretch at succinimide and the $\mathrm{C}=\mathrm{O}$ stretch of the ester, is found to be essentially insensitive to temperature, which is also apparent from the absence of the plateau at large waiting times.

Minimal thermal sensitivity of the mode at $1819 \mathrm{~cm}^{-1}$ helps separating the through-bond energy transport from the through-solvent transport that occurs in quasi-equilibrium conditions. The crosspeak data involving the reporter modes with different thermal sensitivity and the data for mixtures of compounds permitted concluding that through-bond energy transport is the dominant mechanism for the NN/1819 cross-peak data in all four compounds. The through-bond energy transport time, evaluated as the waiting time at which the cross peak maximum is reached, was found to be linearly dependent on the chain length for chain lengths up to $60 \AA$ (Fig. 3A), suggesting a ballistic energy transport regime [7]. The through-bond energy transport speed determined from the chain-length dependence of $T_{\max }$ is found to be ca. $500 \mathrm{~m} / \mathrm{s}$.

The amplitude of the cross peak at $T_{\max }$ reports on the amount of the excess energy delivered from the initially excited mode to the reporter mode. Careful measurements were performed targeting the absolute cross peak amplitudes in different compounds taken at the same concentration within 5\%. The cross-peak amplitude at the maximum is found to decay exponentially with the chain length with a characteristic decay distance of $15.7 \pm 1 \AA$ [8], supporting the ballistic regime.


Fig. 2. Waiting-time dependences for the $\mathrm{N} \equiv \mathrm{N} / 1742 \mathrm{~cm}^{-1}$ (thin line) and $\mathrm{N} \equiv \mathrm{N} / 1778 \mathrm{~cm}^{-1}$ (circles) cross peaks (left) and for the $\mathrm{N} \equiv \mathrm{N} / 1819 \mathrm{~cm}^{-1}$ cross peak (right) for all four compounds in chloroform.

The ballistic transport regime requires to have delocalized vibrational modes. The level of delocalization decreases in a coiled conformation due to disorder induced by structural inhomogeneity and due to chain-to-chain and chain-to-solvent interactions. To evaluate how the chain-to-chain interactions affect the delocalization level we performed DFT normal-mode analysis for azPEG4 in a randomly selected but strongly coiled structure. A single parameter, the degree of delocalization, was calculated for each mode according to the following procedure. The atoms of the PEG chain in azPEG4 were divided into five groups: each of the four repeating units formed a group and the two remaining $\mathrm{CH}_{2}$ groups at the succinimide ester side and the carbonyl group of the ester 
$\left(\mathrm{CH}_{2} \mathrm{CH}_{2} \mathrm{C}=\mathrm{O}\right)$ formed the fifth group. The distribution of atomic displacements for a mode among these five groups was used to determine the degree of the mode delocalization [8]. The delocalization factor, $\gamma$, represents the number of sites a mode is delocalized over: a mode fully delocalized over the PEG chain has $\gamma \sim 5$; the localized modes have $\gamma$ close to unity, while $\gamma=0$ is assigned to modes localized mostly outside of the five groups (Fig. 3B).

Several types of vibrational motion result in delocalized modes; four such high-frequency groups are labeled in Fig. 3B. These groups involve $\mathrm{CH}_{2}$ scissoring $\left(1503-1553 \mathrm{~cm}^{-1}\right.$, bandwidth $\left.50 \mathrm{~cm}^{-1}\right)$, $\mathrm{CH}_{2}$ wagging $\left(1366-1456 \mathrm{~cm}^{-1}\right.$, bandwidth $\left.90 \mathrm{~cm}^{-1}\right), \mathrm{CH}_{2}$ twisting $\left(1246-1340 \mathrm{~cm}^{-1}\right.$, bandwidth $\left.94 \mathrm{~cm}^{-1}\right)$, and C-O, C-C stretching with $\mathrm{CH}_{2}$ rocking $\left(781-1167 \mathrm{~cm}^{-1}\right.$, bandwidth $\left.386 \mathrm{~cm}^{-1}\right)$ groups. The average delocalization factor for each group is determined to be 1.9, 2.6, 2.5, and 2.8, respectively. The deformation modes in the frequency region below $580 \mathrm{~cm}^{-1}$ are found to be most delocalized with the mean delocalization factor of 3.2. The delocalization factor of 3.2 corresponds to a distance of ca. $14.3 \AA$, which is close to the measured characteristic decay distance of $15.7 \AA$.

The ballistic transport is described as a propagation of a vibrational wavepacket having a meanfree-path length of $15.7 \AA$. The experiments were performed in several solvents and in a solid phase. The findings have a potential for developing new efficient signal transduction strategies for molecular electronics and biochemistry.
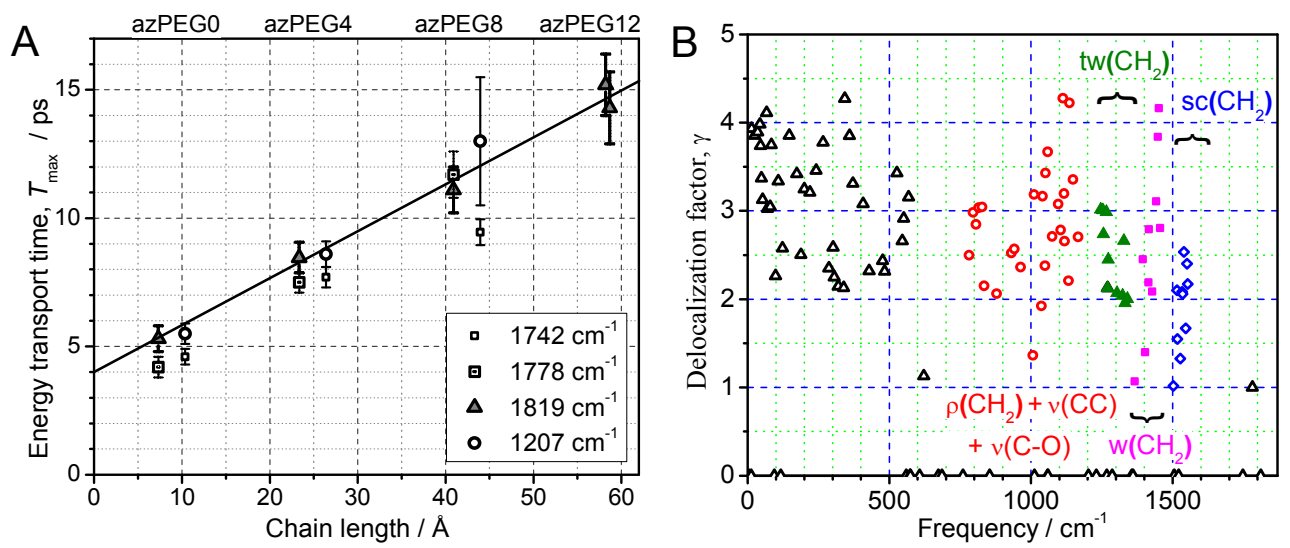

Fig. 3. A. Energy transport time, $T_{\max }$, as a function of through-bond distance. B. The delocalization factor, $\gamma$, computed for azPEG4 is plotted as plotted as a function of mode frequency (harmonic) for all modes below $2000 \mathrm{~cm}^{-1}$. Different groups of modes, including $\mathrm{CH}_{2}$ scissoring (sc), $\mathrm{CH}_{2}$ wagging (w), $\mathrm{CH}_{2}$ twisting (tw), $\mathrm{CH}_{2}$ rocking $(\rho)$, and $\mathrm{C}-\mathrm{C}, \mathrm{C}-\mathrm{O}$ stretching $(\mathrm{v})$ are denoted with different symbols.

Acknowledgement. Support by the National Science Foundation (CHE-0750415) and the Air Force Office of Scientific Research (FA9550-10-1-0007) is gratefully acknowledged.

1. W. Holmes, J. M. Gildemeister, P. L. Richards, and V. Kotsubo, Appl. Phys. Lett. 72, 2250 (1998).

2. C. Yu, L. Shi, Z. Yao, D. Li, and A. Majumdar, Nano Lett. 5, 1842 (2005).

3. D. Schwarzer, P. Kutne, C. Schroeder, and J. Troe, J. Chem. Phys. 121, 1754 (2004).

4. E. H. G. Backus, R. Bloem, R. Pfister, A. Moretto, M. Crisma, C. Toniolo, and P. Hamm, J. Phys. Chem. B 113, 13405 (2009).

5. Z. Wang, J. A. Carter, A. Lagutchev, Y. K. Koh, N.-H. Seong, D. G. Cahill, and D. D. Dlott, Science 317, 787 (2007).

6. D. V. Kurochkin, S. G. Naraharisetty, and I. V. Rubtsov, Proc. Natl. Acad. Sci. U.S.A. 104, 14209 (2007).

7. Z. Lin, and I. V. Rubtsov, Proc. Natl. Acad. Sci. U.S.A. 109, 1413 (2012).

8. Z. Lin, B. Bendiak, and I. V. Rubtsov, Phys. Chem. Chem. Phys. 14, 6179 (2012). 